\title{
Doing less and less: Continuing evolution towards a less invasive approach to infected pancreatic necrosis
}

\author{
Sudeep R. Shah
}

(C) Association of Surgeons of India 2010

Infected pancreatic necrosis is a life threatening complication of acute pancreatitis which is best managed by removal of the infected tissue [1]. Traditionally infected pancreatic necrosis has been subjected to open surgical necrosectomy [2-4].This surgery is associated with high morbidity and significant mortality. Though specialized centers report a mortality below $15 \%$ for infected pancreatic necrosis, this is difficult to achieve and requires extensive utilization of resources and prolonged stay in specialized intensive care units at a high cost to the patient or health care provider.

The treatment of pancreatic necrosis has undergone a gradual evolution over the past 30 years. With increasing experience it also became clear that delaying the surgical intervention in a relatively stable patient with infected pancreatic necrosis improved surgical outcomes. A solitary randomized control trial for early versus late intervention [5] clearly demonstrated this and a delay beyond 3 weeks was endorsed in the International Association of Pancreatology (IAP) guidelines [1].

Two main techniques were employed for surgery- the 'open' [3] technique, wherein repeated debridement were serially performed and the 'closed' approach wherein multiple drains were placed in the cavity following necrosectomy and lavage was instituted [2]. Recent evidence favours the closed, less aggressive approach in view of a lesser incidence of local complications [6]. Thus, even in open surgery, there has been an increasing trend to do less, later, in fewer patients.

\section{S. R. Shah $(\bowtie)$}

Division of GI Surgery

PD Hinduja Hospital, Mumbai 400 016, India

E-mail: dr_sshah@hindujahospital.com
The realization that laparostoma, or open abdomen, was not essential to surgical success, led to the development of minimally invasive approaches. The successes of the laparoscopic approach in selected cases [7] proved that this technique was feasible. At the same time, attempts were made at transgastric endoscopic debridement of the necrosis cavity [8] as well as percutaneous CT guided approaches at cavity drainage [9] with subsequent elective necrosectomy.

The understanding that performing a lesser procedure in a patient often in multi-organ failure has equivalent if not better outcome has increased the use of minimal access procedures for the treatment of infected pancreatic necrosis. Level 1 evidence for the benefit of this approach is now available in the results of the PANTER trial [10] published in the New England Journal of Medicine earlier this year. This well constructed randomized controlled trial compared the traditional approach of open surgical necrosectomy with percutaneous retroperitoneal drainage, followed, if required, by video assisted retroperitoneal debridement (VARD). The results of this study showed that the less invasive stages approach provided significant benefit in the composite primary endpoint of mortality and major complications. These conclusions result from a well conducted, well monitored multi-center study with clearly defined inclusion criteria, including a median delay of over 29 days from the onset of pancreatitis. Only half the patients were referrals to tertiary care centres, the rest being handled at the district level.

In this well designed study, certain points must be highlighted. Patients with abdominal compartment syndrome were excluded. This is perhaps a good indication for open abdomen to reduce compartment pressure and preserve renal and gut perfusion. Secondly, the endpoint was a composite one. Of the two components, mortality was not significantly different between the two groups. However, what was significantly different was organ failure in the peri-operative period. With open surgery, $42 \%$ developed new onset or- 
gan dysfunction in the immediate post-operative period in contrast to only $12 \%$ in the VARD group. This is in spite of the surgery being performed on a relatively stable group of patients wherein less than half were in ICU at the time of surgery and only $36 \%$ had a $>50 \%$ extent of necrosis. This is a clear indicator that the benefit of the study may be a direct result of the gentle multi- step approach preventing deterioration in already sick patients, with upto $35 \%$ avoiding surgery entirely [10].

Long term benefits were also apparent. There was a three time reduction in incidence of incisional hernias with the VARD approach, which is easy to explain owing to the smaller muscle cutting incision. There was also a five fold reduction in need for pancreatic enzyme supplementation and half the incidence of new onset diabetes post procedure, indicating a degree of parenchyma preservation. This indicates that when VARD is performed, a more conservative debridement is done as compared to the open laparotomy approach, without harming outcome.

In the accompanying editorial [11], Warshaw mentions that centers of excellence in North America achieve a low mortality and shorter stay with the traditional approach. However, we must realize that such resources are not available to all. This study has particular relevance to Indian surgeons dealing with infected pancreatic necrosis in setups without the same level of ICU care available in the West. The step up approach is easy to perform as most centers would have a sonologist capable of placing a catheter under sonographic or CT guidance. Further, once the tract has formed, a small incision needs to be taken following this tube, which leads directly to the necrotic tissue. A laparoscope needs to be placed for vision and simple debridement carried out with laparoscopic forceps, following which the wound can be closed over a drain. This relatively simple procedure can be performed without making the patient sicker and taxing the often limited resources of intensive care units.

A shift towards this minimal access step up approach may go a long way to improving the care of this difficult to manage group of patients with reduced morbidity in both the short and long term.

\section{References}

1. Uhl W, Warshaw A, Imrie C, et al. (2002) IAP Guidelines for the Surgical Management of Acute Pancreatitis. Pancreatology 2:565-73

2. Beger HG, Buchler M, Bittner R, Block S, Nevalainen T, Roscher R (1988) Necrosectomy and postoperative local lavage in necrotizing pancreatitis. Br J Surg75:207-12

3. Sarr MG, Nagorney DM, Mucha P, Jr., Farnell MB, Johnson CD (1991) Acute necrotizing pancreatitis: management by planned, staged pancreatic necrosectomy/debridement and delayed primary wound closure over drains. Br J Surg 78:576-81

4. Farkas G, Marton J, Mandi Y, Szederkenyi E (1996) Surgical strategy and management of infected pancreatic necrosis. Br J Surg 83:930-933

5. Mier J, Leon EL, Castillo A, Robledo F, Blanco R (1997) Early versus late necrosectomy in severe necrotizing pancreatitis. Am J Surg173:71-75

6. Heinrich S, Schafer M, Rousson V, Clavien PA (2006) Evidence-based treatment of acute pancreatitis: a look at established paradigms. Ann Surg 243:154-168

7. Cuschieri SA, Jakimowicz JJ, Stultiens G (1998) Laparoscopic infracolic approach for complications of acute pancreatitis. Semin Laparosc Surg 5:189-194

8. Delhaye M, Matos C, Deviere J (2004) Endoscopic technique for the management of pancreatitis and its complications. Best Pract Res Clin Gastroenterol 18:155-181

9. Freeny PC, Hauptmann E, Althaus SJ, Traverso LW, Sinanan M (1998) Percutaneous CT-guided catheter drainage of infected acute necrotizing pancreatitis: techniques and results. AJR Am J Roentgenol 170:969-975

10. van Santvoort HC, Besselink MG, Bakker OJ, et al (2010) A step-up approach or open necrosectomy for necrotizing pancreatitis. N Engl J Med 362:1491-502

11. Warshaw AL (2010) Improving the treatment of necrotizing pancreatitis - a step up. N Engl J Med 362:1535-1537 